\title{
artigo
}

\section{Métodos de rastreamento da ansiedade e depressão em estudantes universitários: revisão integrativa}

\author{
Anxiety and depression tracking methods in college students: integrative review \\ Métodos de seguimiento de ansiedad y depresión en estudiantes universitarios: revisión integradora
}

\begin{abstract}
RESUMO
Objetivo: A presente pesquisa teve como objetivo realizar uma revisão integrativa da literatura de instrumentos utilizados no rastreamento de depressão, ansiedade e misto (ambos) em amostras de estudantes de graduação. Método: a pesquisa ocorreu na base de dados PubMed, de 2009 até 2019. Resultados: Inicialmente foram encontrados 895 artigos, dos quais 345 foram incluídos após a leitura dos títulos e resumos; destes, 316 foram recuperados e posteriormente 176 foram excluídos após a leitura na íntegra, totalizando 140 artigos. Conclusão: Os instrumentos mais utilizados foram: a) Ansiedade: Beck Anxiety Inventory (BAI) ( $n=19$ ) e State-Trait Anxiety Inventory (STAI) ( $n=17$ ); b) Depressão: Beck Depression Inventory (BDI) ( $n=35)$; e c) Misto: Depression, Anxiety and Stress Scale (DASS-21) ( $n=40$ ) e Hospital Anxiety and Depression Scale (HADS) ( $n=19)$. 0 curso mais avaliado foi Medicina e os países com mais produções de artigos foram China $(n=24)$ e Estados Unidos $(n=20)$. 0 Brasil publicou apenas 7 artigos.
\end{abstract}

DESCRITORES: Estudantes; Saúde Mental; Transtornos Mentais.

\section{ABSTRACT}

Objective: The present research aimed to carry out an integrative review of the literature of instruments used to track depression, anxiety and both in samples of undergraduate students. Method: the search took place in PubMed database, from 2009 to 2019. Results: Initially, 895 articles were found, of which 345 were included after reading the titles and abstracts; of these, 316 were recovered and 176 were later deleted after reading in full, totaling 140 articles. Conclusion: The most used instruments were: a) Anxiety: Beck Anxiety Inventory (BAI) $(n=19)$ and State-Trait Anxiety Inventory (STAI) $(n=17)$; b) Depression: Beck Depression Inventory (BDI) ( $n=35)$; and c) Mixed: Depression, Anxiety and Stress Scale (DASS-21) ( $n=40)$ and Hospital Anxiety and Depression Scale (HADS) $(n=19)$. The most evaluated course was Medicine and the countries with the most articles were China $(n=24)$ and the United States $(n=20)$. Brazil published only 7 articles.

DESCRIPTORS: Students; Mental Health; Mental Disorders.

\section{RESUMEN}

Objetivo: La presente investigación tuvo como objetivo realizar una revisión integradora de la literatura de los instrumentos utilizados para el seguimiento de la depresión, ansiedad y mixtos (ambos) en muestras de estudiantes de pregrado. Método: la búsqueda se realizó en la base de datos PubMed, de 2009 a 2019. Resultados: Inicialmente se encontraron 895 artículos, de los cuales 345 fueron incluidos luego de la lectura de títulos y resúmenes; de estos, 316 fueron recuperados y 176 fueron borrados luego de su lectura completa, totalizando 140 artículos. Conclusión: Los instrumentos más utilizados fueron: a) Ansiedad: Inventario de Ansiedad de Beck (BAI) ( $n=19$ ) e Inventario de Ansiedad Rasgo-Estado (STAI) ( $n=17)$; b) Depresión: Inventario de depresión de Beck (BDI) ( $n=35)$; yc) Mixta: Escala de Depresión, Ansiedad y Estrés (DASS-21) ( $n=40)$ y Escala Hospitalaria de Ansiedad y Depresión (HADS) $(n=19)$. El curso más evaluado fue Medicina y los países con más artículos fueron China $(n=24)$ y Estados Unidos $(n=20)$. Brasil publicó solo 7 artículos..

DESCRIPTORES: Estudiantes; Salud Mental; Transtornos Mentales.

RECEBIDO EM: 31/10/2020 APROVADO EM: 16/11/2020

\section{Maira Gabriela Paetzold}

Farmacêutica (UNIOESTE). Mestranda do Programa de Pós-Graduação em Ciências Farmacêuticas (UNIOESTE).

ORCID: 0000-0002-1473-369X 


\section{Ligiane de Lourdes Silva}

Farmacêutica (UNOPAR). Docente assistente no curso de Farmácia e Residências Farmacêuticas na Universidade Estadual do Oeste do Paraná (Cascavel). Doutoranda em Educação na UNIOESTE.

ORCID: 0000-0002-5701-6893

\section{Márcia Regina Simões}

Engenheira Química (UEM). Docente do Centro de Engenharias e Ciências Exatas (CECE) da UNIOESTE. ORCID: 0000-0001-9896-0234

\section{INTRODUÇÃO}

A saúde mental está intimamente relacionada com a saúde física, sendo que as doenças físicas e perturbações mentais resultam da interação de diversos fatores biológicos, psicológicos e sociais. $\mathrm{O}$ estado de psicopatologia interfere nos sistemas endócrino e imunitário, aumentando a suscetibilidade a doenças físicas ${ }^{1}$.

A população jovem é amplamente acometida por transtornos mentais comuns, como depressão, ansiedade e estresse, principalmente estudantes universitários ${ }^{2}$. O ingresso em uma universidade exige dos alunos diversos recursos cognitivos e emocionais para lidar com as demandas do novo ambiente que se encontram ${ }^{3}$. Algumas características podem desencadear processos de estresse nos estudantes universitários, desde a prova de vestibular, de caráter fortemente competitivo; as metodologias de ensino-aprendizagem diferentes daquelas utilizadas no ensino médio, a escolha da especialidade, os longos períodos de aula e a quantidade de informações novas .

No ano de 2015 estima-se que 322 milhões de pessoas sofriam de depressão, o equivalente a $4,4 \%$ da população mundial, e esse número vem aumentando, especialmente em países de baixa renda. $\mathrm{Da}$ mesma forma, estima-se que 264 milhões de pessoas tinham ansiedade, em torno de 3,6\% da população mundial, e assim como a depressão, são mais comuns no sexo femininos.

Em nível mundial, existem diversos estudos de saúde mental em universitários, entretanto, grande parte deles é feita por meio de levantamento de dados em unidades de serviço de saúde mental disponibilizados pelas universidades ${ }^{6}$ ou são pesquisas epidemiológicas realizadas com populações muito específicas, principalmente em estudantes de medici$n^{4,7,8}$. Diante desse contexto, o objetivo dessa pesquisa foi realizar uma revisão integrativa da literatura científica mundial a respeito dos instrumentos de rastreamento de depressão, ansiedade e/ou estresse mais utilizados em estudantes de graduação.

\section{MÉTODOS}

Realizou-se uma revisão integrativa de acordo com as seis fases do processo de elaboração de revisão integrativa 9,10 e o método PRISMA foi utilizado para auxiliar o desenvolvimento dessa revisão integrativa. A pergunta norteadora foi: Quais são as escalas ou instrumentos mais utilizados para avaliar sintomas de ansiedade, depressão e/ou estresse em estudantes de graduação?

A base de dados utilizada foi a PubMed e a busca foi realizada no dia 15 de outubro de 2019. Todos os descritores utilizados estavam na língua inglesa e foram escolhidos de acordo com o interesse da busca, utilizando os operadores booleanos AND e OR. Dessa forma, os termos selecionados foram anxiety OR anxiety disorder OR anxiety symptoms OR anxiety state AND depression OR depressive disorder OR depressive syndrome OR depressive symptoms AND scale OR instrument OR asessment tool AND adults AND undergraduate OR student. A busca incluiu artigos apenas na língua inglesa, publicados até outubro de 2019, não sendo aplicado limite temporal.

A coleta de dados ocorreu entre os dias 15 e 29 de outubro de 2019. Os critérios de inclusão foram: trabalhos científicos que utilizavam escalas para o rastreamento de depressão, ansiedade e/ ou estresse e amostras que continham estudantes de graduação ou pós-graduação. Foram excluídos artigos com amostras de não estudantes, crianças e estudantes do ensino médio.

A busca foi realizada por uma pesquisadora e validada por outras duas pesquisadoras. Os artigos selecionados foram exportados para a plataforma Mendeley, onde aqueles que atendiam aos critérios de inclusão foram selecionados e armazenados, de acordo com a leitura do título e resumo. Após a recuperação e leitura dos artigos na íntegra, foram excluídos artigos publicados antes de 2009 , aqueles com amostras que não eram exclusivas de estudantes de graduação e os que possuíam diferentes intervenções. Por fim, foram incluídos apenas os artigos publicados entre 2009 e 2019, com amostras exclusivas de estudantes de graduação maiores de 18 anos e sem uso de intervenções.

Os artigos incluídos ao final do processo tiveram suas informações compiladas na planilha do Excel, onde as variáveis analisadas foram: instrumentos utilizados, área de estudo, país de realização da pesquisa e desenho do estudo.

\section{RESULTADOS}

Inicialmente foram encontrados 895 artigos na PubMed e após a leitura dos títulos e resumos, 550 foram excluídos por apresentarem amostras de não estudantes, crianças e/ou adolescentes. De 345 artigos restantes, 316 foram recuperados e lidos na íntegra. Foram excluídos 176 artigos por terem sido publicados antes de 2009, ou por terem amostras não exclusivas de estudantes de graduação e aqueles com intervenções; perfazendo um total de 140 artigos para a etapa final. 


\section{Figura 1. Fluxograma dos estudos selecionados para a revisão integrativa}

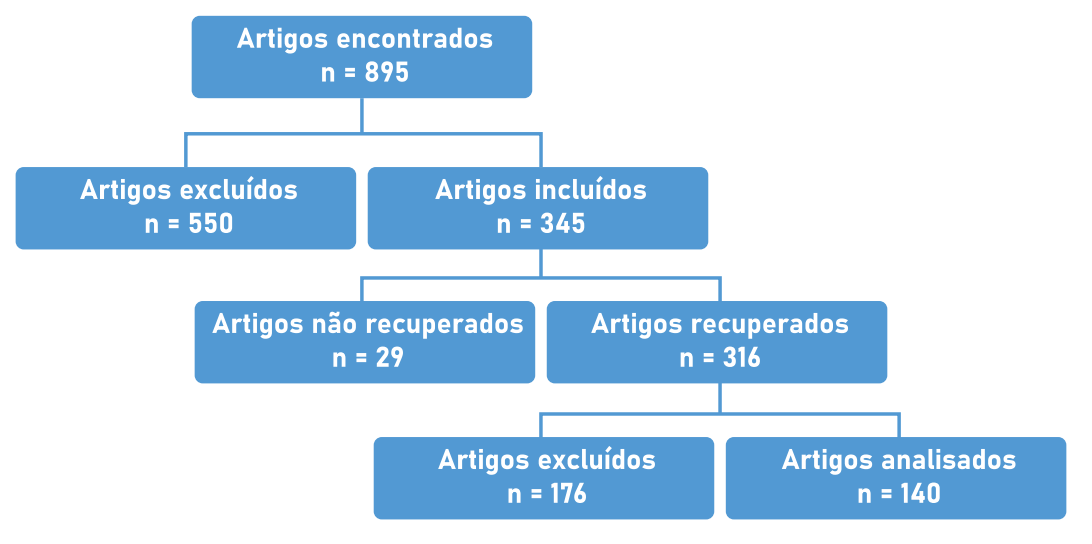

Fonte: autor (2020)

Tabela 1. Instrumentos de Avaliação de Ansiedade, Depressão e Misto utilizados em estudantes de graduação.

$$
\text { Instrumentos }
$$

Ansiedade

Beck Anxiety Inventory (BAI)

$19 \quad 13,57$

State Trait Anxiety Inventory (STAI)

17

Generalized Anxiety Disorder 7 (GAD-7)

Self-rating Anxiety Scale (SAS)

Kuwait University Anxiety Scale (KUAS)

Hamilton Anxiety Rating Scale (HAMA)

Generalized Anxiety Disorder 2 (GAD-2)

Situational Anxiety Scale

The Anxiety Symptom Questionnaire

Depressão

Beck Depression Inventory I e II (BDI)

3525,00

Center for Epidemiologic Studies-Depression Scale (CES-D)

Patient Health Questionnaire 9 (PHQ-9)

Self-Rating Depression scale (SDS)

Patient Health Questionnaire 2 (PHQ-2)

Hamilton Rating Scale for evaluation of depression (HAMD)

Depressive Experiences Questionnaire (DEQ)

Adolescent Depression Inventory (ADI)

Misto

Depression, Anxiety and Stress Scale (DASS-21)

$40 \quad 28,57$

Hospital Anxiety and Depression Scale (HADS)

The symptom check-list 90-R (SCL-90-R)

General Health Questionnaire 28 (GHQ-28)

General Health Questionnaire 12 (GHQ-12)

$4 \quad 2,86$

Patient Health Questionnaire 4 (PHQ-4)
O fluxograma das etapas pode ser visualizado na Figura 1.

Os instrumentos encontrados nos artigos incluídos no estudo foram divididos em três categorias: os que avaliaram somente ansiedade, os que avaliaram somente depressão e instrumentos mistos, que avaliaram, dentre outros distúrbios, ansiedade e depressão. Na Tabela 1 observam-se todos os instrumentos encontrados.

Dentre 140 artigos, 79 (56,43\%) não especificaram qual a área ou o curso dos estudantes; e dentre aqueles que especificaram, foi possível encontrar 25 áreas de estudo. Representando a maioria, 43 $(30,71 \%)$ artigos avaliaram a saúde mental do curso de Medicina, e outros cursos, como Psicologia e Odontologia foram avaliados em sete (5\%) artigos e Farmácia e Enfermagem, em 5 (3,57\%) artigos.

Ao total, 41 países foram encontrados, dos cinco continentes. $\mathrm{O}$ continente que mais produziu artigos foi a Ásia $(n=68$; $48,57 \%$ ), com destaque para a China, com $24(17,14 \%)$ produções. Em seguida, Europa e América, com a produção de 38 $(27,14 \%)$ e $36(25,71 \%)$ artigos, respectivamente. Dentre esses dois continentes, destacam-se Turquia, com 12 (8,57\%) artigos; e os Estados Unidos da América, com 20 (14,29\%) produções.

Os diferentes desenhos de estudo também foram avaliados nessa revisão integrativa. A maioria $(\mathrm{n}=82,58,57 \%)$ dos trabalhos realizou estudos transversais, $54(38,57 \%)$ dos artigos não especificou o tipo de desenho utilizado; e estudos coorte, longitudinais e caso-controle foram utilizados por dois (1,43\%), $1(0,71 \%) \mathrm{e}$ um $(0,71 \%)$ dos autores, respectivamente.

\section{DISCUSSÃO}

Por meio da elaboração dessa revisão integrativa foi possível encontrar diversos instrumentos de rastreamento de ansiedade e depressão utilizados em amostras de estudantes de graduação, em todos os continentes, por diversas populações. O grande número de artigos encontrados pode ser devido à facilidade e conveniência de utilizar uma amostra de estudantes, com 


\begin{tabular}{|c|c|c|}
\hline The Mood and Anxiety Symptom Questionnaire e Short Form (MASQ-SF) & 1 & 0,71 \\
\hline Chinese College Student Mental Health Scale & 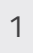 & 0,71 \\
\hline $\begin{array}{l}\text { Depression and Generalized Anxiety versions of the Mini International } \\
\text { Neuropsychiatric Interview (MINI) }\end{array}$ & 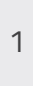 & 0,7 \\
\hline Anxiety and Depression subscales of the Brief Symptom Inventory (BSI) & 1 & 0,71 \\
\hline The Hopkins Symptoms Checklist (HSCL-25) & 1 & 0,71 \\
\hline Composite International Diagnostic Interview Screening Scales (CIDI-SC) & 1 & 0,71 \\
\hline Aga Khan University Anxiety and Depression Scale (AKUADS) & 1 & 0,7 \\
\hline Mood and Anxiety Symptom Questionnaire-90 (MASQ-90) & 1 & 0,7 \\
\hline autor (2020) & & \\
\hline
\end{tabular}

a população da própria Universidade em que é realizado o estudo ${ }^{11}$. Outro fato que pode justificar o interesse de autores em avaliar a saúde mental de estudantes universitários é a faixa etária em que a maioria se encontra, pois jovens com menos de 21 anos tem maior risco de desenvolvimento de desordens mentais ${ }^{12}$.

Apesar da grande quantidade de artigos recuperados, poucas pesquisas foram realizadas no Brasil (5\%), o que pode ser justificado pelo fato de que o volume de publicações de estudos voltados para avaliação da saúde mental no Brasil não pode ser considerado crescente ${ }^{11}$.

Nas pesquisas realizadas no Brasil, foram utilizados dois instrumentos de ansiedade, um instrumento de depressão e dois instrumentos mistos. Foram eles: Beck Anxiety Inventory (BAI) e State Trait Anxiety Inventory (STAI); Beck Depression Inventory (BDI); e Hospital Anxiety and Depression Scale (HADS) e Depression, Anxiety and Stress Scale (DASS).

Os instrumentos utilizados para rastreamento da ansiedade e depressão foram validados em suas versões brasileiras. $\mathrm{O}$ Beck Anxiety Inventory (BAI) foi validado e apresentou adequada confiabilidade, com a de Cronbach de 0,87 para população de universitários ${ }^{13}$. Da mesma forma, o State Trait Anxiety Inventory (STAI) também foi validado e mostrou elevada consistência interna, com $\alpha$ de Cronbach de 0,85 na escala "Estado" e de 0,88 na escala "Traço" ${ }^{14}$. O questionário Beck Depression Inventory (BDI) na sua versão brasileira foi validado em uma amostra de estudantes universitárias, apresentando $\alpha$ de Cronbach de 0,89 ${ }^{15}$.
Apesar da grande quantidade de artigos recuperados, poucas pesquisas

foram realizadas

no Brasil (5\%),

o que pode ser justificado pelo fato de que o volume de publicações de estudos voltados

para avaliação da

saúde mental no

Brasil não pode

ser considerado

crescente.
Em relação aos instrumentos mistos, suas versões brasileiras também foram validadas. A Depression, Anxiety and Stress Scale (DASS) apresentou valores de $\alpha$ de Cronbach de 0,92 para a escala de depressão, 0,90 para a escala de estresse e 0,86 para a escala de ansiedade ${ }^{16}$; e a Hospital Anxiety and Depression Scale (HADS) foi somente validada em amostras de pacientes, apresentando valores de $\alpha$ de Cronbach de 0,68 para a escala de ansiedade e 0,77 para a escala de depressão ${ }^{17}$.

Algumas semelhanças podem ser apontadas em relação a uma revisão sistemática realizada em 2019, onde o instrumento encontrado pelos autores como o mais utilizado para avaliação da depressão foi o Depression, Anxiety and Stress Scale (DASS), encontrado em 11 estudos, de um total de 34 analisados $^{18}$. O presente estudo mostrou que a mesma escala foi o instrumento misto mais utilizado nas pesquisas, encontrado em 40 dos 140 artigos analisados. Da mesma forma, em outra pesquisa ${ }^{11} \mathrm{o}$ instrumento encontrado mais utilizado para a avaliação da depressão foi o Beck Depression Inventory (BDI), encontrado em 105 artigos, dentre 175 avaliados. Esse dado pode ser comparado com os resultados encontrados nessa pesquisa, que mostram o mesmo instrumento como o mais utilizado dentre aqueles que somente avaliam a depressão.

Grande parte dos artigos prestou atenção especial ao curso de Medicina e outros cursos da saúde. Isso pode ser justificado devido ao contato direto de agentes da saúde com pacientes, seus familiares, suas dificuldades e superações. A influência dos pacientes sobre o estado mental dos profissionais pode ser um dos motivos pelo qual os pesquisadores se preocupam tanto com a saúde mental de agentes da saúde, em especial médicos. A responsabilidade dos profissionais da saúde sobre a qualidade de vida e saúde de outras pessoas também pode ser um fator estressante para essa classe.

O presente estudo possui algumas limitações, como a utilização de apenas uma base de dados. Por esse motivo, os resultados devem ser analisados com cautela, pois podem existir outros artigos em ou- 


\section{artigo}

tras bases de dados com resultados distintos. Outra limitação é a inclusão de artigos em apenas um idioma, de maneira que os resultados de artigos publicados em outros idiomas podem apresentar resultados diferentes dos encontrados nessa revisão integrativa. Dessa forma, sugerimos que novas pesquisas sejam realizadas com mais bases de dados, por mais pesquisadores e em diversos idiomas.

\section{CONCLUSÕES}

É possível concluir que muitos países, em especial a China e os Estados Unidos da América realizam pesquisas a respeito da avaliação da saúde mental de seus estudantes, excepcionalmente da área de Medicina. Nesses estudos foram encontrados variados instrumentos para a avaliação da ansiedade e depressão, sendo os mais encontrados a De- pression, Anxiety and Stress Scale (DASS) e o Beck Depression Inventory (BDI). Grande parte dos artigos encontrados não avaliou as propriedades psicométricas dos instrumentos utilizados, dados que poderiam fornecer valiosas informações sobre a estrutura dos questionários. Deste modo, encorajamos novos estudos que utilizem amostras de diferentes estudantes e que avaliem as características psicométricas desses instrumentos.

\section{REFERÊNCIAS}

1. Tavares FL et al. Mortalidade por suicídio no Espírito Santo, Brasil: análise do período de 2012 a 2016. Av Enferm. 2020; 38 (1):66-76.

2. Ribeiro DB, Terra MG, Soccol KLS, Schneider JF, Camillo LA, Plein FAS. Motivos da tentativa de suicídio expressos por homens usuários de álcool e outras drogas. Rev Gaúcha Enferm. 2016 mar;37(1):e54896. doi: http://dx.doi. org/10.1590/19831447.2016.01.54896.

3. FélixT., OliveiraE., LopesM. V., ParenteJ. R., DiasM. S., \& MoreiraR. M. (2016). Fatores de risco para tentativa de suicídio: Produção de conhecimento no Brasil. Revista Contexto \& Saúde, 16(31), 173-185. https://doi.org/10.21527/21767114.2016.31.173-185.

4. Assumpção GLS, Oliveira LA, Souza MFS de. Depressão e suicídio: uma correlação. Rev. Pret. [Internet]. $7^{\circ}$ de março de 2018 [citado $10^{\circ}$ de novembro de 2020];3(5):312-33. Disponível em: http://periodicos.pucminas.br/index.php/pretextos/article/ view/15973.

5. Muller Alcantara Sônia, et al. Estratégias de prevenção e pósvenção do suicídio: Estudo com profissionais de um Centro de Atenção Psicossocial. Revista de Psicologia, IMED, 2017; 9 (2): 6 23. http://dx.doi.org/10.18256/2175-5027.2017.v9i2.1686.

6. Borba LO, Ferreira ACZ, Kalinke LP, Maftum MA, Maftum GJ. Fatores associados à tentativa de suicídio por pessoas com transtorno mental. Rev Min Enferm. 2020; 24: e-1284.

7. Pessoa DMS, Freitas RJM, Melo JAL, Barreto FA, Melo KCO, Dias ECS. Assistência de enfermagem na atenção primária à saúde de adolescentes com ideações suicidas. Rev Min Enferm. 2020; 24: e-1290.

8. Palma DCA, Santos ES, Ignotti E. Análise dos padrões espaciais e caracterização dos suicídios no Brasil entre 1990 e 2015. Cad. Saúde Pública 2020; 36(4): e00092819.

9. Vasconcelos Neto PJA, Moreira RS, Oliveira Junior FJM, Ludermir AB. Tentativa de suicídio, transtorno de estresse pós-traumático e fatores associados em mulheres do Recife. Rev Bras Epidemiol 2020; 23: E200010.

10. Bahia CA, Avanci JQ, Pinto LW, Minayo MCS. Notificações e internações por lesão autoprovocada em adolescentes no Brasil, 2007-2016. Epidemiol. Serv. Saude 2020; 29(2): e2019060,2020.
11. SIVVA - Sistema de Informação e Vigilância de Violências e Acidentes. [base de dados na internet]. São Paulo: Acidentes de Trânsito. [acesso em 23 de agosto de 2020]. Disponível em: http://www.prefeitura.sp.gov.br/ secretarias/saude.

12. Santos LA, Kind L. Integralidade, intersetorialidade e cuidado em saúde: caminhos para se enfrentar o suicídio Interface (Botucatu). 2020; 24: e190116.

13. Fernandes FY et al. Tendência de suicídio em adolescentes brasileiros entre 1997 e 2016 . Epidemiol. Serv. Saude 2020; 29(4): e2020117.

14. Almeida ABM, Uchoa GF, Carvalho AMR. Epidemiologia das intoxicações medicamentosas registradas no sistema nacional de informações tóxico-farmacológicas de 2012-2016. Saúde e Pesqui. 2020; 13(2): 431-440.

15. Franck MC, Monteiro MG, Limberger RP. Mortalidade por suicídio no Rio Grande do Sul: uma análise transversal dos casos de 2017 e 2018. Epidemiol. Serv. Saude 2020, 29(2): e2019512.

16. Fernandes DAA, Ferreira NS, Castro JGD. Perfil epidemiológico das tentativas de suicídio em Palmas-Tocantins, de 2010 a 2014. Tempus, actas de saúde colet 2016, 10(4), 09-23.

17. Matos CCSA, Tourinho FSV. Saúde da População Negra: como nascem, vivem e morrem os indivíduos pretos e pardos em Florianópolis (SC). Rev Bras Med Fam Comunidade 2018; 13(40):113.

18. Cordeiro EL, Silva LSR, Mendes EWP, Silva LCL, Duarte VL, Lima ECMP. Tentativa de suicídio e fatores associados ao padrão uso e abuso do álcool. Rev. Eletrônica Saúde Mental Álcool Drog 2020; 16(1): 1-10.

19. Grigoletto AP, Souto VT, Terra MG, Tissot GL, Ferreira CN. Tentativas de suicídio notificadas em um hospital de ensino no estado do Rio Grande do Sul, 2014-2016. R. pesq.: cuid. fundam. online 2020; 12: 413-419.

20. Rodrigues MF, Oliveira PP, Silva HC, Pinheiro JMC. Comportamento suicida: o perfil epidemiológico das lesões autoprovocadas no estado de Goiás. Rev Cient Esc Estadual Saúde Pública Goiás "Cândido Santiago" 2020; 6 (2): e600003.

21. Santos Junior CJ, Santos IV, Silva JVS, Gomes VM, Ribeiro MC. Perfil de pacientes atendidos por tentativa de suicídio em um Hospital Geral de Emergências do estado de Alagoas, Brasil. Medicina (Ribeirão Preto. Online) 2019; 52 (3):223-230. 